Conclusion: This is the first study to examine mediation and highlights the importance of potentially modifiable targets for HPRs to reduce mortality in older adults with osteoarthritis. Encouraging people to maintain levels of physical activity at a population level and targeting a reduction in physical limitation in clinical practice to allow this, is important.

Disclosure of Interests: None declared

DOI: 10.1136/annrheumdis-2019-eular.5862

\section{OP0156-HPR THE FEASIBILITY AND EFFECTIVENESS OF TELEMEDICINE FOR KNEE OSTEOARTHRITIS IN DISEASE MANAGEMENT: A RANDOMISED CONTROL TRIAL}

Zhengping Huang ${ }^{1}$, Xiaoyan Zhong ${ }^{2}$, Zhimin Xie ${ }^{2}$, Ll. Tianwang ${ }^{1} .{ }^{1}$ Guangdong Second Provincial General Hospital, Guangzhou, China; ${ }^{2}$ Guangdong Online Hospital, Guangzhou, China

Background: Osteoarthritis $(O A)$ is a serious health concern worldwide, and patients with $O A$ are in urgent need of proper long-term disease management to prevent disability and improve the quality of life. Research has started to investigate the feasibility and effectiveness of telemedicine in providing disease management for patients with chronic diseases. Guangdong Online Hospital $(\mathrm{GOH})$ is the first officially recognized web-based hospital that widely provides telemedicine services in southern China.

Objectives: This study aimed to establish the feasibility and effectiveness of $\mathrm{GOH}$ in providing long-term disease management for patients with knee OA via a 6-month, randomised control trial (RCT).

Methods: 40 patients with knee OA were randomly assigned to receive conventional therapy $(\mathrm{CT})$ in the clinic or conventional therapy plus a brief $\mathrm{GOH}$-based intervention (CT-GOHT). GOH-based intervention included educational lectures, medical suggestions and psychotherapy. The primary outcome was the drop-out rates and the secondary outcomes included the mean change of the self-reported total score of the Western Ontario and McMaster Universities Osteoarthritis Index (WOMAC), the Multidimensional Fatigue Inventory (MFI), the Hospital Anxiety and Depression Scale (HADS) and the Pittsburgh Sleep Quality Index (PSQI) from baseline to endpoint.

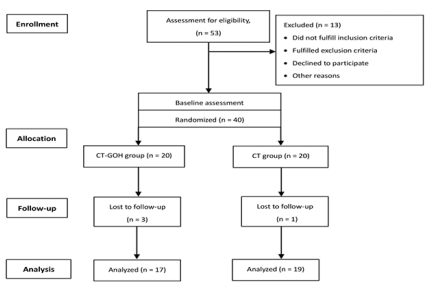

Figure 1. Flow chat of trial participation

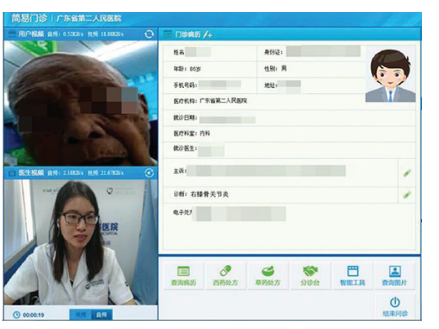

Figure 2. A telemedicine intervention to patients with knee $O A$

Results: The baseline characteristics were comparable between the two groups. 3 patients in CT group and 1 patient in CT-GOHT group lost to follow-up during the study $(P=0.598)$. A statistically significant difference was observed in the mean change of WOMAC recommended index of joint pain $(8.82 \pm 5.02 \mathrm{vs} .13 .90$ $\pm 7.63, P=0.026)$, morning stiffness (3.12 \pm 1.50 vs. $4.42 \pm 2.06, P=0.039$ ), functional limitation $(27.65 \pm 9.91$ vs. $38.84 \pm 17.28, P=0.025)$, HADS-Anxiety ( $3.24 \pm$ 2.41 vs. $5.32 \pm 3.23, P=0.037)$, and PSQI global score ( $2.35 \pm 1.54$ vs. $4.21 \pm$ 3.34, $P=0.043$ ) from baseline to endpoint when comparing the CT group to the CT-GOHT group. In addition, Guangdong Online Hospital was widely accepted by the including OA patients for their disease management.

Conclusion: Delivering a telemedicine intervention to patients with knee OA via $\mathrm{GOH}$ is a feasible and potentially effective method in long-term disease management, especially for patients living in remote areas. The results provide preliminary experiences and guidance for an upcoming full-scale RCT in disease management via telemedicine.
Trial registration: ChiCTR1800014465.

\section{REFERENCE:}

[1] Huang Z, et al. Implementation of telemedicine for knee osteoarthritis: study protocol for a randomized controlled trial. Trials, 2018, 19(1):232

Table 1. Baseline characteristics of the two study groups

\begin{tabular}{lcc}
\hline Variable & $\begin{array}{c}\text { CT group } \\
(\mathrm{n}=20)\end{array}$ & $\begin{array}{c}\text { CT-GOH group } \\
(\mathrm{n}=20)\end{array}$ \\
\hline Age & $72.25 \pm 8.84$ & $67.25 \pm 10.97$ \\
Female, $\mathrm{n}$ & 15 & 15 \\
Education, $\mathrm{n}$ & 12 & 14 \\
Primary school & 8 & 6 \\
High school & & \\
Han Chinese, $\mathrm{n}$ & 20 & 20 \\
Income, $\mathrm{n}$ & 8 & 5 \\
<¥2000 & 10 & 13 \\
$¥ 2000$ - $¥ 5000$ & 2 & 2 \\
> $¥ 5000$ & $25.85 \pm 3.87$ & $26.29 \pm 2.96$ \\
BMI, kg/m ${ }^{2}$ & $105.83 \pm 100.35$ & $111.33 \pm 92.54$ \\
Distance to hospital, kilometre & 8 & 6 \\
Kellgren-Lawrence grade, $\mathrm{n}$ & 10 & 13 \\
Grade I & 2 & 1 \\
Grade II & & $21.00 \pm 8.38$ \\
Grade III & $18.90 \pm 10.08$ & $9.25 \pm 4.68$ \\
WOMAC pain, 0-10 & $8.45 \pm 4.89$ & $63.30 \pm 25.31$ \\
WOMAC morning stiffness, 0-10 & $58.05 \pm 20.55$ & $45.70 \pm 20.43$ \\
WOMAC function, 0-10 & $43.55 \pm 19.77$ & $8.30 \pm 4.64$ \\
MIF & $8.90 \pm 5.55$ & $6.85 \pm 4.55$ \\
HADS-A & $7.10 \pm 5.28$ & $7.70 \pm 5.13$ \\
HADS-D & $7.35 \pm 4.56$ & \\
PSQI global score & &
\end{tabular}

Table 2. Mean change of outcome measures from baseline to endpoint

\begin{tabular}{lccc}
\hline Results & CT group $(n=17)$, & CT-GOH group, $(n=19)$ & $P$ value \\
\hline WOMAC pain & $8.82 \pm 5.02$ & $13.90 \pm 7.63$ & 0.026 \\
WOMAC morning stiffness & $3.12 \pm 1.50$ & $4.42 \pm 2.06$ & 0.039 \\
WOMAC function & $27.65 \pm 9.91$ & $38.84 \pm 17.28$ & 0.025 \\
MIF & $17.53 \pm 7.73$ & $20.95 \pm 8.75$ & 0.225 \\
HADS-A & $3.24 \pm 2.41$ & $5.32 \pm 3.23$ & 0.037 \\
HADS-D & $2.12 \pm 2.06$ & $2.36 \pm 2.01$ & 0.714 \\
PSQI global score & $2.35 \pm 1.54$ & $4.21 \pm 3.34$ & 0.043 \\
\hline
\end{tabular}

Disclosure of Interests: None declared

DOI: 10.1136/annrheumdis-2019-eular.6474

\section{OP0157-HPR EUROPEAN PATIENT VOICE IN GOUT SURVEY - SUBJECTIVE SATISFACTION IN GOUT PATIENTS VERSUS OBJECTIVE SUBOPTIMAL GOUT CARE}

Marc De Meulemeester ${ }^{1}$, Tim Jansen ${ }^{2}$, Gudula Petersen ${ }^{3}$, Fernando Perez-Ruiz ${ }^{4}$. ${ }^{1}$ MEDIF sprl, Gozée, Belgium; ${ }^{2}$ VieCuri Medisch Centrum, Venlo, Netherlands; ${ }^{3}$ Grünenthal GmbH, Aachen, Germany; ${ }^{4}$ Hospital de Cruces, Barakaldo, Spain

Background: Gout is the most common form of inflammatory arthritis with an increasing prevalence and incidence in many countries (1). Few information is available about current management of gout and satisfaction of patients living with gout.

Objectives: To evaluate diagnosis and treatment of gout from patient perspective to improve treatment outcomes for gout patients in Europe.

Methods: A 15 minute online survey with gout patients from 14 European countries (Austria, Belgium, France, Denmark, Germany, Italy, Ireland, Malta, Netherlands, Norway, Portugal, Spain, Sweden, Switzerland) was conducted in 2018. The design and content of the survey has been developed together with several patient and clinical experts

Results: 1,100 gout patients ( $78 \%$ males, mean age 55 years) with a mean number of 2.9 flares in the past 12 months completed the survey. $77 \%$ percentage of patients are suffering from co-morbidities (hypertension $52 \%$, dyslipidemia $41 \%$, overweight $40 \%$, type 2 diabetes $23 \%$, long-term illness of kidney/renal insufficiency $17 \%$ ). It was reported that mainly general practitioners are involved in the diagnosis of the disease $(73 \%)$, discussion of gout $(68 \%)$ and treatment of last gout flare $(59 \%)$, whereas rheumatologists are less involved in diagnosis $(8 \%)$, discussion (15\%) and treatment (11\%) of last gout flare. $22 \%$ of patients were not diagnosed until they have had 4 or more flares. $71 \%$ of patients had flares in the past 12 months (thereof $33 \% \geq 3$ flares) which seemed to be particularly an issue for younger patients. $58 \%$ of patients receive uric acid lowering therapies, $43 \%$ pain killers, $25 \%$ colchicine, $13 \%$ non-medical pain relief and $12 \%$ corticosteroids. $59 \%$ of patients do not have regular follow up appointments for their gout and $53 \%$ 